\author{
Military Technical College \\ Kobry El-Kobbah, \\ Cairo, Egypt.
}

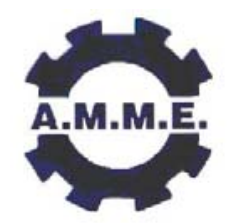

\title{
NUMERICAL SIMULATION OF AN IN-LINE TUBE BUNDLE USING THE URANS APPROACH
}

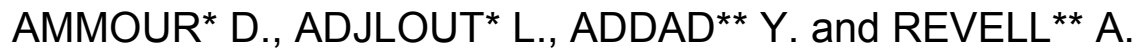

\begin{abstract}
In the present work, a turbulent flow across a square in-line tube bundle is computed using the Unsteady Reynolds Averaged Navier-Stokes (URANS) approach. The pitch ratio used in the tube bundle configuration is $P / D=T / D=1.44$ and the Reynolds number is 70000 based on tube diameter. Both $2 \mathrm{D}$ and $3 \mathrm{D}$ computational domains are considered. Turbulence models tested include the standard $k-\varepsilon$ model of Jones et al. [12], the $k-\omega$ Shear Stress Transport (SST) model of Menter [10] and the Reynolds Stress Model of Speziale et al. [11] (SSG). In addition, the recently developed, SST- $C_{\text {as }}$ model by Revell [1] is also tested. The unstructured industrial code Code-Saturne has been used for the present study. In general, the flow predictions using the 2D grid fail to capture correctly the general flow physics; while on the other hand, the 3D calculations give predictions of pressure fields and velocity profiles that are in broad agreement with both LES results [2] and the more recent experimental data [4]. With regards to turbulence models, a comparison of the pressure coefficient and velocity profiles revealed that the SST- $C_{\text {as }}$ and RSM models, both seem to give reasonable predictions of the flow, capturing the asymmetric behaviour of the flow as the reference data. As expected, the standard $K-\varepsilon$ model fails to capture this behaviour and predicts a rather symmetric flow field. The pressure spectra from the SST- $C_{\text {as }}$ calculation indicates a single clear peak at around the frequency $45 \mathrm{~Hz}(\mathrm{St}=0.84)$, similar to that observed in LES predictions. This suggests that both large and small re-circulations coexist on the wake of centre tubes resulting in the shear stress to be higher in the bottom than on the top of the tubes.
\end{abstract}

\section{KEY WORDS}

Tube bundles, RANS, URANS, turbulence modelling, asymmetric flow.

* Faculty of Mechanical Engineering, Department of Marine Engineering University of Science and Technology U.S.T.ORAN-MB, B.P.1505 Oran El Mnaouar 31000, Oran, ALGERIA.

** School of MACE, Manchester University, M60 1QD, Manchester, UK. 


\section{NOMENCLATURE}

\section{Latin symbols}

\begin{tabular}{|c|c|}
\hline$A$ & Instantaneous variable \\
\hline$A^{*}$ & Temporal averaged variable \\
\hline $\bar{A}$ & Averaged variable \\
\hline$\widetilde{A}$ & Solved variable \\
\hline$a^{\prime}$ & Instantaneous fluctuation \\
\hline$a^{\prime \prime}$ & Residual fluctuation \\
\hline$a_{1}$ & Coefficient of the SST model $a_{1}=0.31$ \\
\hline$C_{a s}$ & Degree of alignment between stresses and strains \\
\hline$C_{p}$ & Pressure coefficient \\
\hline$F_{2}$ & Second blending function for the SST model \\
\hline$N$ & Number of periods \\
\hline$P$ & Pressure \\
\hline$P^{*}$ & Temporal Averaged pressure \\
\hline $\bar{P}$ & Averaged pressure \\
\hline$\widetilde{P}$ & Solved Pressure \\
\hline$\|S\|$ & Filtered strain rate magnitude \\
\hline$S_{i j}$ & Strain tensor \\
\hline$S_{t}$ & Strouhal number \\
\hline$T_{1}$ & Period of the phenomenon \\
\hline$t$ & Time \\
\hline$\overline{U_{i}}$ & Averaged velocity \\
\hline$U_{i}^{*}$ & Temporal Averaged velocity \\
\hline$\widetilde{U}_{i}$ & Solved velocity \\
\hline$u_{i}$ & Component of velocity according to "x" \\
\hline$u_{j}$ & Component of velocity according to "y" \\
\hline$u_{i}^{\prime \prime}$ & Residual velocity fluctuation \\
\hline$u_{0}$ & Inlet velocity \\
\hline$x_{i}$ & Coordinate \\
\hline$x_{j}$ & Coordinate \\
\hline
\end{tabular}

\section{Greek symbols}
$\varepsilon$
Isotropic dissipation
Turbulent kinetic energy
Turbulent viscosity
$v_{t}$
Density
$\omega$
Dissipation rate per unit of kinetic energy 


\section{INTRODUCTION}

The fluid flow across tube bundle configurations results in a complex unsteady coupling from one tube to the next. In practical situations, this motion leads to random excitation forces and can cause low-amplitude tube oscillations which can result in long-term fretting-wear or fatigue. For heat exchangers, the flow would certainly have a direct effect on the heat transfer rate and thus a direct impact on the equipment efficiency. Due to these issues and to its direct practical relevance to industrial applications, the flow across tube bundles has been the subject of numerous experimental and numerical studies.

Aiba et al. [3] conducted an experimental study on square in-line tube banks for gap ratio 1.2 and 1.6. It has been observed that the tube response of the downstream cylinders is quite different from the upstream ones. The pressure distribution around the cylinder surface exhibits a highly deflected flow with a stagnation point of around 45 degrees. The flow behaviour is asymmetric in both these configurations, which is thought to be due to the narrow gap ratios.

In parallel to the present study, Afgan [2] conducted numerical simulations of flow across square in-line tube bundles using a commercial code (Star-CD). A range of RANS models have been tested in addition to the LES simulations using fine grids. It has been found that the 2D RANS predictions were unable to capture correctly the flow, and so extensive 3D LES were performed for all gap ratios. For example, Rollet-Miet et al. [6] presented the first LES calculations of the turbulent flow across a staggered tube bundle type of configuration using a finite-element code. Hassan and Barsamian [7] performed 3D LES of the turbulent flow across a five row staggered tube bundle with some success including the prediction of turbulent levels. More recently, Benhamadouche et al. [8] performed a 3D LES of the turbulent flow across in-line tube bundles using the industrial finite volume Code_Saturne. They obtained interesting results and found that the selection of the subgrid scale model was not critical for this type of application; due to the fact that the turbulence in the computational domain is mainly fed by large structures generated in the wake region as opposite to bounded flows, with no obstacles or wakes, in which the prediction of the near-wall region plays a major role in the turbulence generation and flow mixing. Liang and Papadakis [9] performed 3D LES simulation with around $700 \mathrm{~K}$ cells of the steady approaching flow over both in-line and staggered tube bundles.

In recent years, the use of URANS to conduct numerical studies of complex unsteady flows has gained popularity within the research community. This is due to the lower computational cost offered by this technique in comparison to the DNS, LES and even hybrid RANS-LES approaches. Undeniably, this makes this approach a very attractive one, especially in cases where only mean values are of interest. In the present work a flow across a square in-line tube bundle (see Fig.1.) is computed using the URANS approach. Results are compared with the LES predictions conducted with a fine grid by Afgan [2] and the recent experimental data of Yahiaoui [4]. Different RANS models are tested in the unsteady mode to examine which of those models is able to mimic the flow physics and reproduce the unsteady behaviour across the tubes. 


\section{GOVERNING EQUATIONS}

The Navier-Stokes equations are the basic governing equations and are defined as:

$$
\frac{\partial u_{i}}{\partial t}+u_{j} \frac{\partial u_{i}}{\partial u_{j}}=-\frac{1}{\rho} \frac{\partial P}{\partial x_{i}}+v \frac{\partial^{2} u_{i}}{\partial x_{j} \partial x_{j}}
$$

\section{Rans}

In a RANS calculation, the generic instantaneous variable is decomposed into two components; the time averaged, $\overline{\mathrm{A}}$ and instantaneous fluctuation, $a^{\prime}=A-\bar{A}$ according to:

$$
\mathrm{A}(\mathrm{x}, \mathrm{t})=\overline{\mathrm{A}}+a^{\prime}(x, t)
$$

The Reynolds Averaged Navier-Stokes equations are thus given as:

$$
\frac{\partial \bar{u}_{i}}{\partial t}+\bar{u}_{j} \frac{\partial \bar{u}_{i}}{\partial x_{j}}=-\frac{1}{\rho} \frac{\partial \bar{P}}{\partial x_{i}}+v \frac{\partial^{2} \bar{u}_{i}}{\partial x_{j} \partial x_{j}}-\frac{\partial \overline{u_{i}^{\prime} u_{j}^{\prime}}}{\partial x_{j}}
$$

\section{Urans}

Initially, the URANS calculations can be defined by the continuity of a RANS calculation in a statically periodic flow, the average is exactly equivalent to an average of phase [5], defined by:

$$
\bar{A}(x, t)=\lim \frac{1}{N+1} \sum_{n=0}^{\infty} A\left(x, t+n T_{1}\right)
$$

Where $T_{1}$ is the period of the phenomenon. The instantaneous variable $A$ is decomposed according to:

$$
A(x, t)=\widetilde{A}(x, t)+a^{\prime \prime}(\mathrm{x}, \mathrm{t})
$$

With by definition:

$\widetilde{\mathrm{A}}=\bar{A} \quad$ is the solved variable.

$a^{\prime \prime}=A-\bar{A} \quad$ is the residual fluctuation.

The variable solved itself is decomposed into a temporal average $\mathrm{A}^{*}$ and a fluctuation a' as: 


$$
\widetilde{A}(x, t)=A^{*}(x)+a^{\prime}(x, t)
$$

Finally, combining replacing equation 6 in 5 results in a triple decomposition:

$$
A(x, t)=A(x)+a^{\prime}(x, t)+a^{\prime \prime}(x, t)
$$

Thus, the pressure and velocity are then decomposed according to:

$$
\begin{aligned}
& U_{i}=U_{i}^{*}+u_{i}{ }^{\prime}+u_{i}{ }^{\prime} \\
& P_{i}=P_{i}^{*}+p_{i}{ }^{\prime}+p_{i}{ }^{\prime \prime}
\end{aligned}
$$

The Unsteady Reynolds Averaged Navier-Stokes equations are:

$$
\frac{\partial \widetilde{U}}{\partial t}+\widetilde{U}_{j} \frac{\partial \widetilde{U}_{i}}{\partial x_{j}}=-\frac{1}{\rho} \frac{\partial \widetilde{P}}{\partial x_{i}}+v \frac{\partial^{2} \widetilde{U}_{i}}{\partial x_{j} \partial x_{j}}-\frac{\partial \overline{u^{\prime \prime}{ }_{i} \mu_{j}}}{\partial x_{j}}
$$

It has to be noted that equations 3 and 10 are similar from an implementation point of view. That is, no extra terms appear in one or the other.

Although, the development of RANS models has been formally based on the assumption that the flow is statistically in steady state, i.e. all the fluctuations are accounted for by the Reynolds stresses, many of the commercial and industrial codes that are used nowadays allow for the computations to be run in unsteady modes until the steady state is reached. However, if the flow is originally unsteady there is an "automatic" switch from equations 3 to 10 . Thus the role of the turbulence model implicitly changes and it has to response now correctly to this flow variation in time.

Various RANS models are implemented in the industrial Code_Saturne. The models tested in the present study are; the standard two-equation hi-Reynolds $k-\varepsilon$ model of Jones et al. [12], the k-w SST turbulence model of Menter [10], the Reynolds stress model of Speziale et al. [11], and the recently developed $C_{a s}$ model of Revell [1].

The $C_{a s}$ model formulation is obtained by small modifications to the SST model. It based on the observation that in contrast to eddy viscosity models, the Reynolds stress model calculates an exact production which is explicitly linear in the mean strain rate. Effectively, one can write exactly $P_{k}=C_{a s} k\|S\|$ where the non-dimensional parameter, $C_{a s}$ represents the degree of alignment between stresses and strains:

$$
C_{a s}=-\frac{a_{i j} S_{i j}}{\|S\|}
$$


$a_{i j}$ is the turbulent stress anisotropy tensor; $S_{i j}$ is the strain rate tensor, and $\|S\|$ is an invariant defined as $\sqrt{2 S_{i j} S_{i j}}$.

The SST model requires only small modifications to incorporate the $C_{a s}$ model. The first is by changing the production rate of turbulence in the $k$ equation, and the second by modifying the formulation of the turbulent eddy viscosity, as follows:

$$
v_{t}=k \min \left(\frac{1}{\omega} ; \frac{a_{1}}{\|S\| F_{2}} ; \frac{C_{a s}}{\|S\|}\right)
$$

The value of $C_{a s}$ in equation (11) is limited to \pm 0.31 , which ensures that the original underlying turbulent viscosity as calculated by the SST model will remain unaltered in regions of equilibrium steady mean flow. When the flow becomes unsteady or there exists a phase lag between the turbulent stresses and the mean strain rates, it is intended that the transport equation for $C_{a s}$ will respond to this, and thus be reduced. This reduction will have the effect to reduce the production of turbulent kinetic energy in a manner equivalent to that observed in a full Reynolds Stress Transport scheme. When the value of $C_{a s}$ becomes negative, translating to a negative value for the production term, the model is effectively transporting energy back to the mean flow equations (Eq. 10) in a similar way to the observed effect known as 'backscatter' in LES. In the diffusion term, the absolute value of $C_{a s}$ is used in order to enable the associated reduction in viscosity to be felt by the mean flow whilst preserving numerical stability.

The SST- $C_{a s}$ model has been developed and tested by Revell $[1,13]$ on various 1D cyclic strains, the fully developed channel, the Oscillating channel, the flow over a NACA0012 aerofoil at $20^{\circ}$ incidence and a circular cylinder in square duct, in which this model has showed a noticeable superiority in the flow prediction over the other twoequation RANS models. The model has also been shown to give improvements in statistically steady turbulent flows with dominant curvature effects, such as the trailing vortex downstream of a wingtip [14].

\section{RESULTS AND DISCUSSIONS}

The present test case requires only periodic and wall boundary conditions (see Fig.1). The computations presented in this section have been conducted at first using a $2 \mathrm{D}$ grid $(21,600$ cells) with a single cell in the spanwise direction, and then the same runs were repeated using a 3D mesh $(604,800$ cells). This second grid was generated by extruding the $2 \mathrm{D}$ mesh in the spanwise direction $(z=2 . \mathrm{D})$. For both grids the nondimensional normal distance of the first cell centre from the wall, $y^{+}$, was kept at values ranging between 13 and 70 (all computations have been carried out using $\mathrm{Hi}-\mathrm{Re}$ models with wall-function). The grids resolution in $\mathrm{x}$ and $\mathrm{y}$; the horizontal and vertical directions respectively, is illustrated in Fig.2. In the following, first the results using 2D grid are presented and discussed then this is followed by the ones using the 3D mesh. 


\section{Runs using the 2D grid}

Figure 3 shows the instantaneous pressure contours in the $x-y$ plan as predicted by the different URANS models. At first glance, all the models, with exception of standard $\mathrm{k}-\varepsilon$ model, seem to predict reasonably well the transient and asymmetric behaviour of the flow. Even though all the computations are carried out in a transient mode, the standard two-equation $\mathrm{k}-\varepsilon$ model is observed to suppress all pressure fluctuations, resulting in a smooth and identical, pressure distribution in all 4 gaps between the cylinders. On the other hand, the RSM model followed by the $C_{a s}$ model then the k- $\omega$ SST model, predicts a much more realistic unsteady motion. For example, the instantaneous pressure field as predicted by the RSM model shows clear zonal lower values of pressure which is an indication of vortices located at these zones. The animation of this numerical simulation reveals that these vortices are generated at the lower part of the cylinders and travel at a given angle through the computational domain, which is most likely to be at the origin of the flow asymmetry.

A more detailed comparison can be made by plotting the predicted profiles of the normalized pressure coefficient, $C_{p}=2\left(\mathrm{P}-\mathrm{P}_{\text {ref }}\right) / \rho U_{0}^{2}$ around the central tube (see Fig.4.) in which the angle, in the abscissa, is defined as illustrated in Fig.1. In addition to the present URANS models predictions, the LES results of Afgan [2] for aspect ratio of 1.5 and the recently made available experimental data of Yahiaoui [4], (for the same gap ratio as the present computations of 1.44) are also included in the figure. Illustrated in Fig.4, the effect of the flow deflection is observed in term of the stagnation pressure region located somewhere around $45^{\circ}$ from the flow direction. All URANS models seems to predict the correct pressure coefficient peak at this location but soon after the angle of $80^{\circ}$, the URANS profiles deviate dramatically from the experimental data set, predicting not only a delayed reattachment point but also a completely erroneous pressure coefficient distribution at angles from $280^{\circ}$ to $360^{\circ}$. As it will be become clear further down, this is meanly due to the computations being carried out in a 2dimensional domain. Indeed, as opposite to steady state computations in which the whole energy spectrum is modeled, the URANS models are thought to capture some of the turbulent structures that may interact and/or stretch in the spanwise direction (see Fig.10) making the symmetry assumption in a two-dimensional domain less justifiable in this periodically unsteady case.

\section{Runs using the 3D grid}

Figure 5 shows the averaged pressure distribution in the $x-y$ plan as obtained from the 3D grid runs. In general, the predictions of all RANS models are observed to be in a reasonably good agreement with the LES data. Also, in accord with the above mentioned observations, the stagnation point is indicated by the peaks of pressure positioned on the top of the tubes at the angle of $45^{\circ}$. On the other hand, the minimum values located in the bottom of tubes are caused by the detachment of shear layer and the recirculation region. The results of the standard two-equation $\mathrm{k}-\varepsilon$ model are omitted from this figure because as mentioned above the model is found unable to capture the flow unsteadiness (the results are identical to the ones shown in Fig.3). 
Figure 6 shows the evolution of normalized pressure coefficient around the central tube. In contrast to the 2D calculations, the URANS profiles now show a better agreement with the LES and experimental data all around the tube. The $C_{a s}$ and the k- $\omega$ SST models are now predicting the correct position of flow reattachment. Furthermore, all models are now found to predict a better distribution of the pressure coefficient at the angles ranging from $280^{\circ}$ to $360^{\circ}$. It is interesting to notice that the URANS models are predicting the peak level of $C_{p}$ in better agreement with the experimental data in comparison to the LES. One possible explanation comes from the fact that this region is dominated by high levels of turbulence compared to the rest of the domain and is thus suggesting that a longer physical time for gathering statistics is needed for the LES computations.

Figure 7 shows the comparison between the predictions of 3D URANS models and LES results [2] of mean velocity distribution in the $x-y$ plan. Again, the figure illustrates that all the models are capable to predict reasonably well the flow deflection, in good agreement with the LES data. It is interesting to notice that the newly developed 3D equations SST- $C_{a s}$ model returns similar flow predictions to the second moment closure with a relatively lower computational cost.

Figure 8 shows the mean velocity profile in the wake of tube at the streamwise position $X=4.33$. With the exception of the $k-\varepsilon$ model, all URANS models capture a maximum peaks of velocity around $U / U o=1$ far from the wall in free stream flow in agreement with the experimental and LES data. The minimum value is also well predicted around zero close to the wall and in the gap space where the viscous forces are localized. All the curves show the same trend but only the experiment [3] and LES results are observed to follow a particular velocity variation in the gap space region $(2<Y / D<3.5)$.

Figure 9 shows fluctuating pressure and its power spectral density (PSD) at a location of probe 6 (the probe location is also illustrated in the figure). The pressure spectra reveal one clear peak around the frequency $45 \mathrm{~Hz}$ corresponding to the Strouhal number of 0.84 . This peak can be associated with the vortex shedding detected in the shear regions.

Figure 10 shows instantaneous $Q$ iso-surfaces computed from the predictions obtained with the SST- $C_{a s}$ model. A large number of worm-type of vortices is observed in the centre of domain. These structures are observed to originate at the cylinders walls and elongate in the same direction as the across flow.

\section{CONCLUSIONS}

- The flow across in-line tube bundles is found to be asymmetric and transient.

- URANS calculations using k- $\omega$ SST, RSM and SST-Cas turbulence models are broadly able to capture the transient flow behaviour, while the $k-\varepsilon$ model suppresses all flow fluctuations. 
- The stagnation point is located somewhere around 45 degrees; in agreement with the LES calculations of Afgan and the experiment recently carried out at The Univeristy of Oran, Algeria.

- From the analysis of pressure and velocity spectra, it is concluded that there is a peak frequency at around $45 \mathrm{~Hz} \quad(S t=0.84)$, in agreement with the LES predictions, implying that the vortex shedding detected in the shear regions with the URANS models is realistic.

- The SST- $C_{a s}$ model is observed to predict the mean flow quantities in good agreement with the RSM model and at a lower computational cost

- Two principle recirculation regions are observed behind the central tube, with one being significantly larger than the other; the larger one is located underneath the tube and the smaller one is above. This results in the shear stress underneath this tube to be higher than that observed above it.

- Finally, good quantitative and qualitative agreement with the experimental results shows the ability of the present approach (Unsteady RANS) to correctly reproduce the essential physics associated with massively separated flow. Especially, the results obtained the SST- $C_{a s}$ model, are very encouraging making the model a good candidates for further investigations. The model can also be further improved in future work by doing a local averaging of the $C_{a s}$ value in a similar manner to the dynamic sub-grid LES models to enhance its numerical stability.

\section{AKNOWLEDGEMENTS}

This work was supported by the EU program Tempus "COFFEE". JEP-31131-2003. Acknowledgements are also expressed Dr. I. Afgan and Dr. J. Uribe for their invaluable guidance and continuous advice. We are also very thankful to Mr. T. Yahiaoui for making the experimental data available.

\section{REFERENCES}

[1] Revell, A., "A Stress-Strain Lag Eddy Viscosity Model for Mean Unsteady Turbulent Flows", PhD thesis submitted to the University of Manchester, UK, (2006).

[2] Afgan, I., "Large Eddy Simulation of Cylindrical Bodies Incorporating Unstructured Finite Volume Mesh", PhD thesis submitted to The University of Manchester, UK, (2007).

[3] Aiba, S., Tsuchida, H., Ota, T. "Heat transfer Around Tubes in In-line Tube Banks", Bull JSME, 25. 919-926; in Flow around circular cylinders Vol 2: Applications by Zdravkovich, M. M, (1982).

[4] Yahiaoui, T., University of USTOran, Algeria, personal communications, (2007). 
[5] Fadai-Ghotbi, A., "Modélisation de la Turbulence en Situation Instationnaire par Approche URANS et hybride RANS-LES. Prise en Compte des Effets de Paroi par Pondération Elliptique", PhD thesis submitted to the University of Poitiers, France, (2007).

[6] Rollet-Miet, P., Laurence, D. R., Ferziger, J., "LES and RANS of Turbulent Flow in Tube Bundles", Int. J. of Heat and Fluid Flow, 20, pp241-254, (1999).

[7] Barsamian, H.R., Y.A., Hassan, "Large Eddy Simulation of Turbulent Cross Flow in Tube Bundles", Nuclear Engineering and Design, 172,103-122, (2004).

[8] Benhamadouche, S., Laurence, D., Jarrin, N., Afgan I., Moulinec, C., "Large Eddy Simulation of Flow Across In-line Tube Bundle", NURETH-11, Avignon, France, (2005).

[9] Liang, C., Papadakis G., "Large Eddy Simulation of Cross Flow over In-line and Staggered Tube Bundles", Flow Induced Vibration, de Langre \& Axisa ed. Ecole Polytechnique, Paris, 6-9th July, (2004).

[10] Menter, F. R., "Zonal Two Equation k- $\omega$ Turbulence Models for Aerodynamic Flows", AIAA Paper 93-2906, (1993).

[11] Speziale, C. G., Sarkar, S., Gatski, T.B., "Modelling the Pressure-strain Correlation of Turbulence: an Invariant Dynamical System Approach", Journal of Fluid Mechanics, pp 245-272, (1991).

[12] Jones, W. P., Launder, B.E., "The Prediction of Laminarization with a TwoEquation Model of Turbulence", Int. J. of Heat Mass Transfer, pp 301-314, (1972).

[13] Revell, A.J., Benhamadouche, S., Craft, T.J., Laurence, D.R., "A Stress-Strain Lag Eddy Viscosity Model for Unsteady Mean Flow", Int. J. Heat Fluid Flow, Vol. 27, pp. 821-830, (2006).

[14] Revell A., Duraisamy K., laccarino G., "Advanced Turbulence Modelling of Wingtip Vortices", Turbulence and Shear Flow Phenomena TSPF5, Munich. Germany. 27 August, (2007). 


\section{FIGURES}

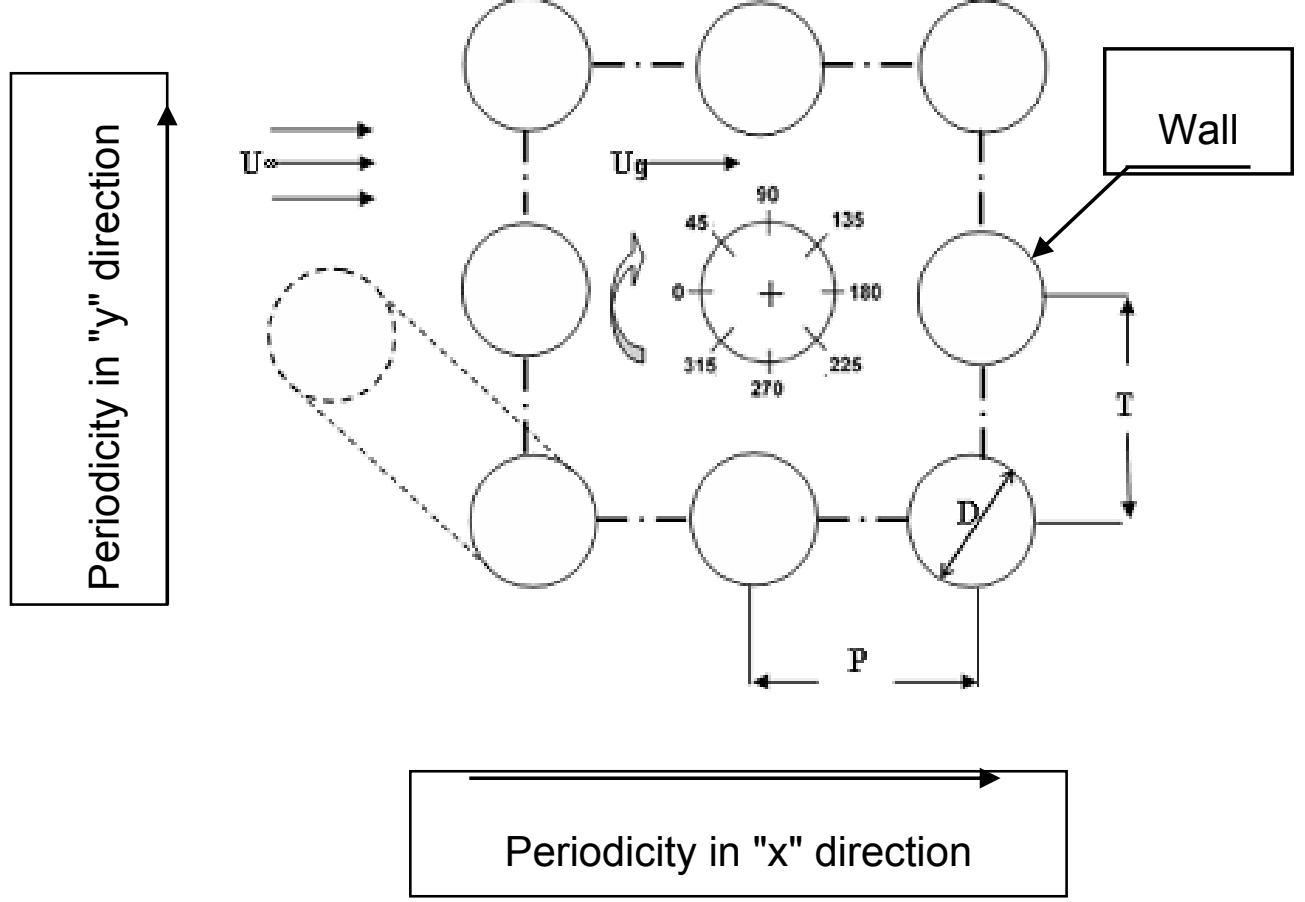

Fig.1. Geometry and boundary conditions of in-line tube bundles.
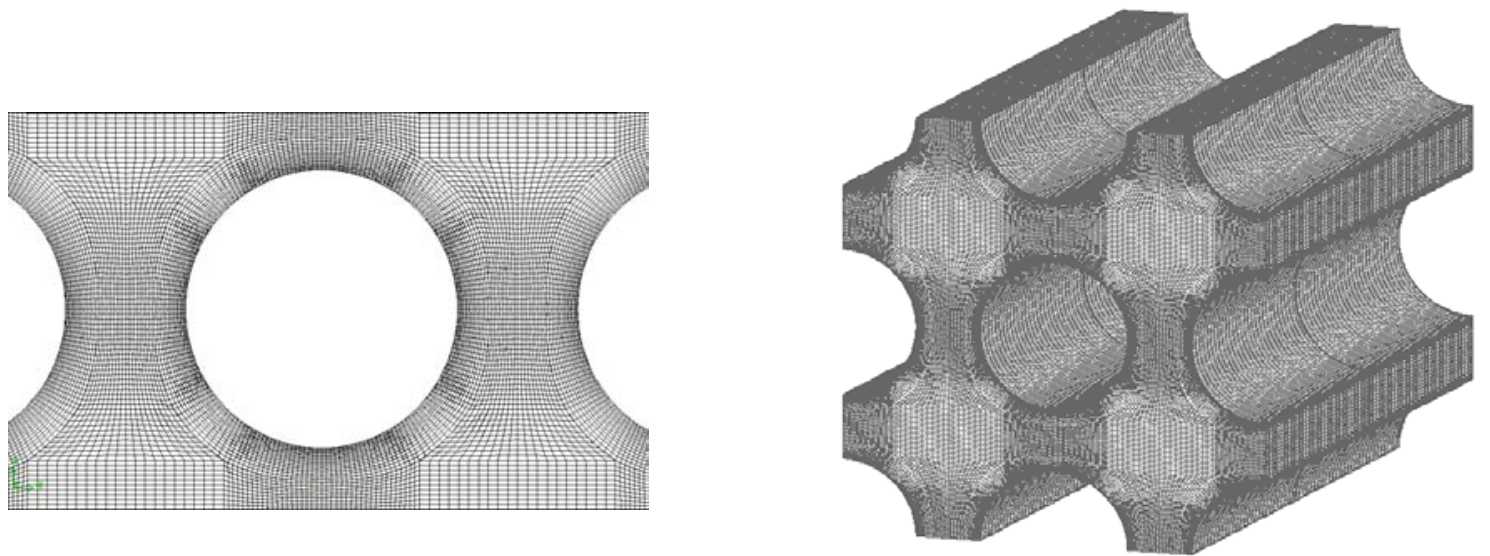

Zoom on the centre of the tube

Fig.2. 2D and 3D grids for the present study. 


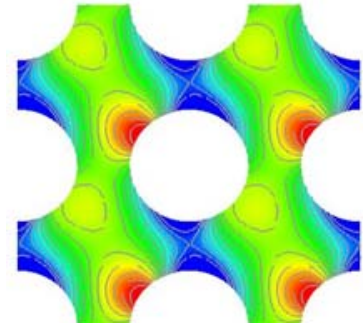

$\mathrm{K}-\varepsilon$

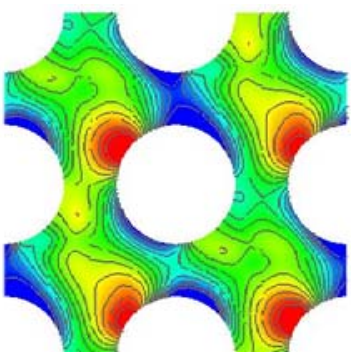

$\mathrm{K}-\omega \mathrm{SST}$

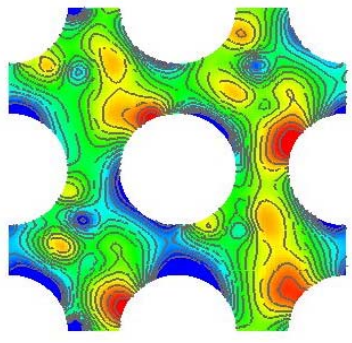

RSM

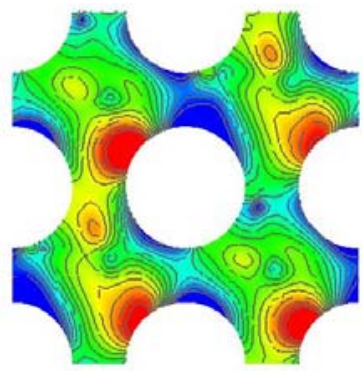

SST- $C_{a s}$

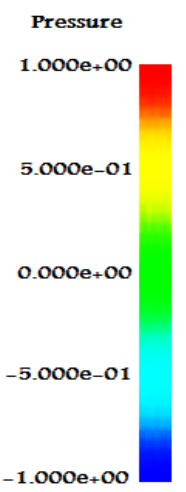

Fig.3. Instantaneous pressure contours in an X-Y cross sectional view.

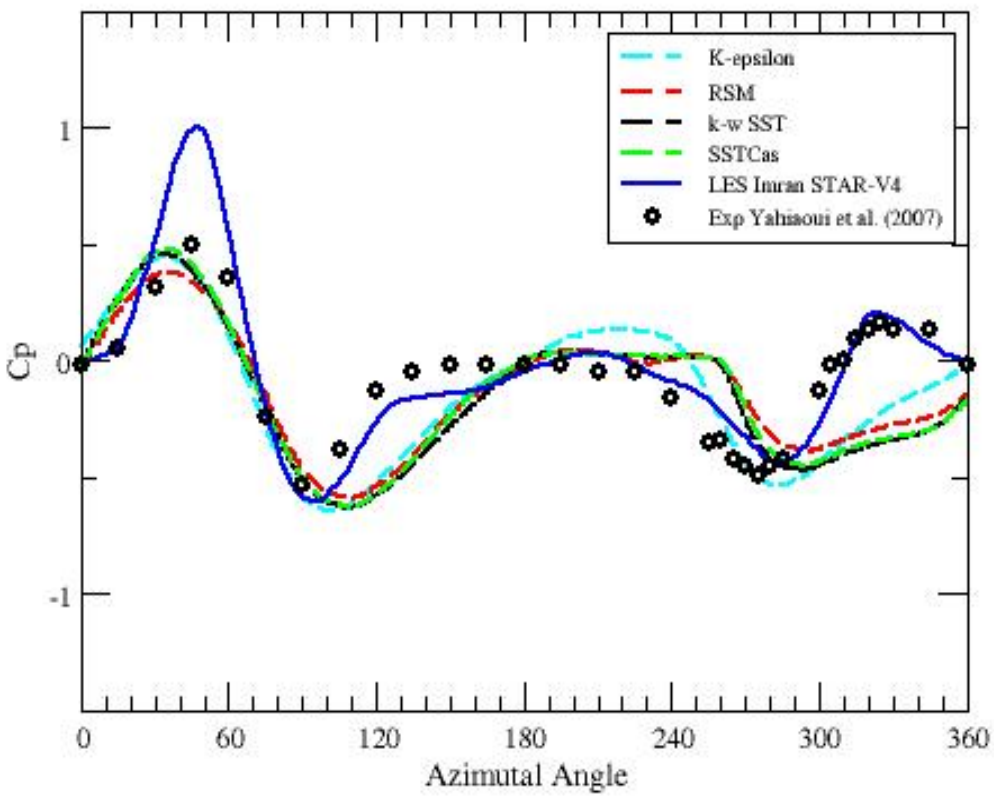

Fig.4. Mean pressure distribution around the centre tube, comparison made between the 2D URANS models, LES and experiment. 


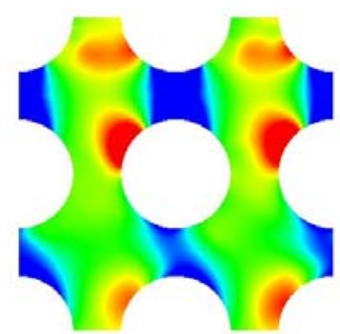

$\mathrm{K}-\omega \mathrm{SST}$

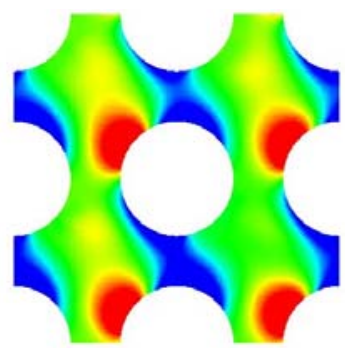

SST- $C_{a s}$

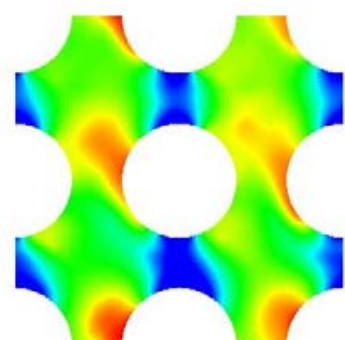

RSM

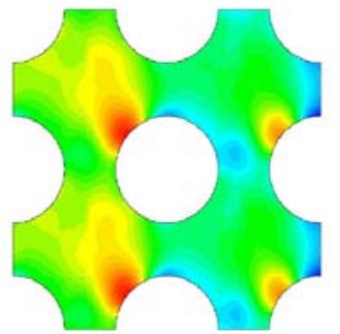

LES (Afgan)

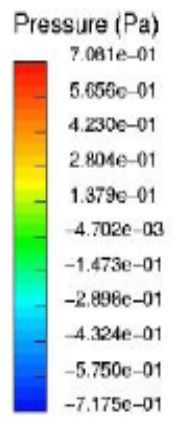

y

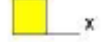

Fig.5. 3D mean pressure distributions in an X-Y cross view.

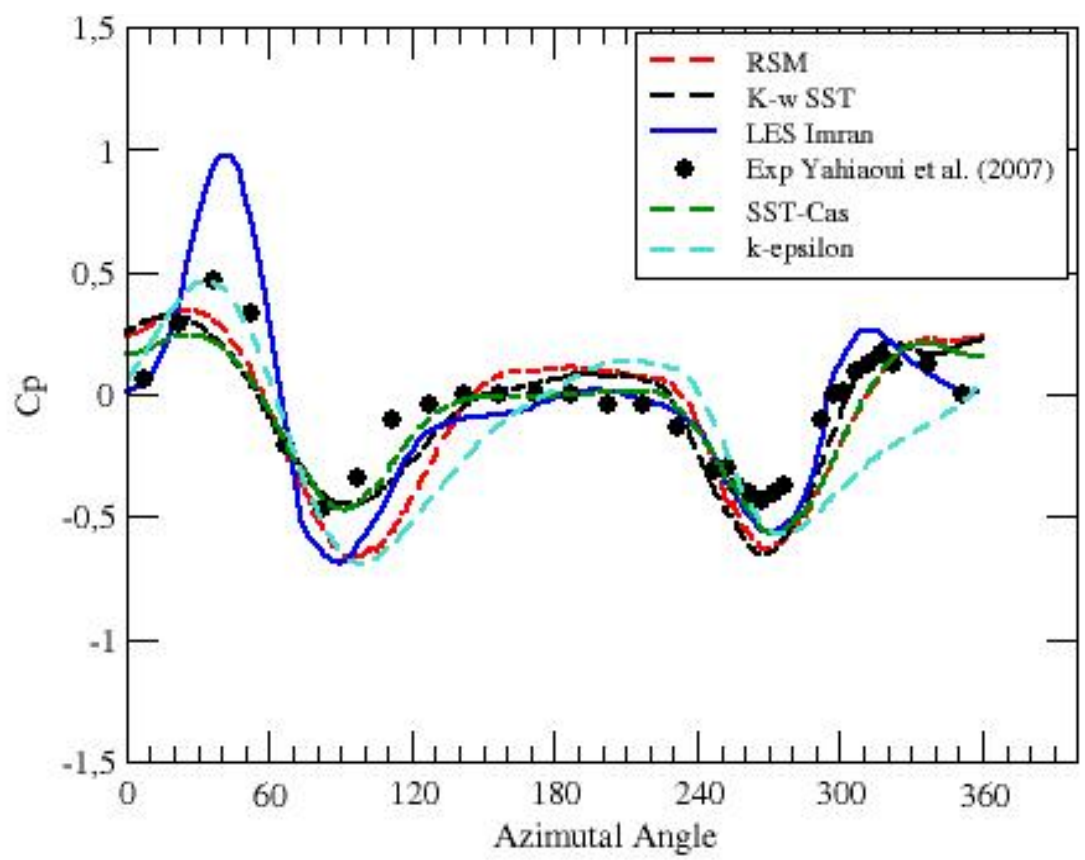

Fig.6. Mean pressure distribution around the centre tube, comparison made between the 3D URANS models, LES and experiment. 


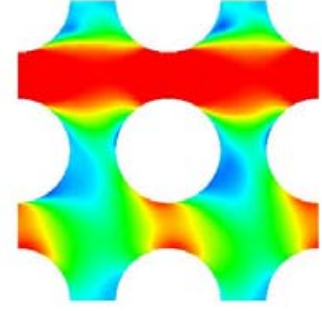

$\mathrm{K}-\omega \mathrm{SST}$

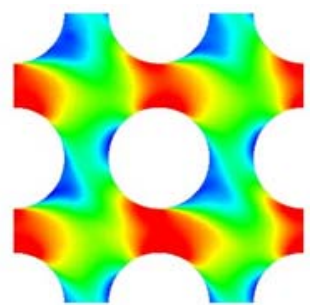

SST- $C_{a s}$

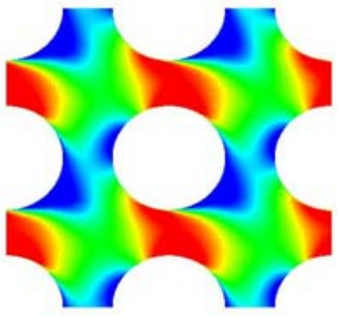

RSM

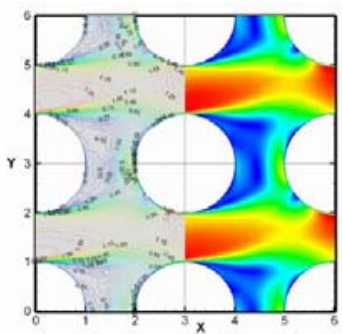

LES (Afgan)
Velocity $(\mathrm{m} / \mathrm{s})$ $1.729 \mathrm{e}+00$ $1.556 \mathrm{c}+00$ $1.384 e+00$ $1.211 \mathrm{e}+00$ $1.038 \mathrm{e}+00$ B. $655 e-01$ 8.929e-01 $5.202 \mathrm{e}-01$ 3.475e-01 $1.749 \mathrm{e}-01$ $2.192 e-03$

y

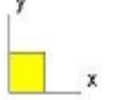

Fig.7. 3D Averaged velocity field in an $X-Y$ cross view.

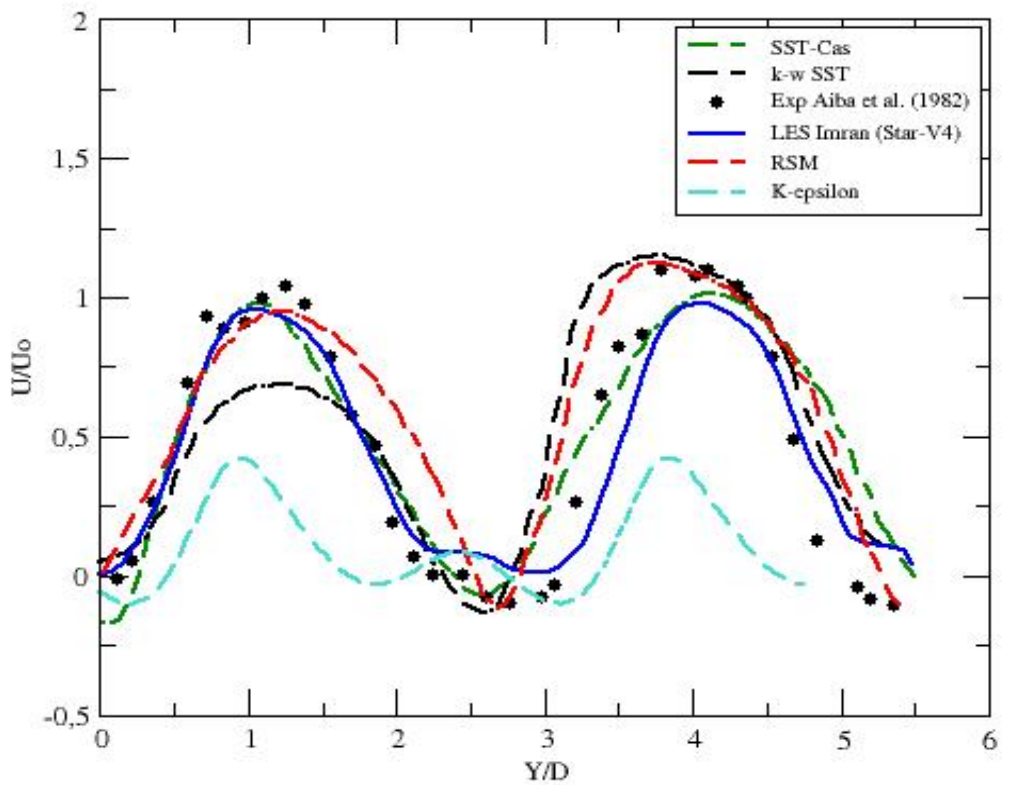

Fig.8. 3D mean velocity along a vertical line in the wake of the central tube at $x=4.33 \mathrm{~cm}$. 

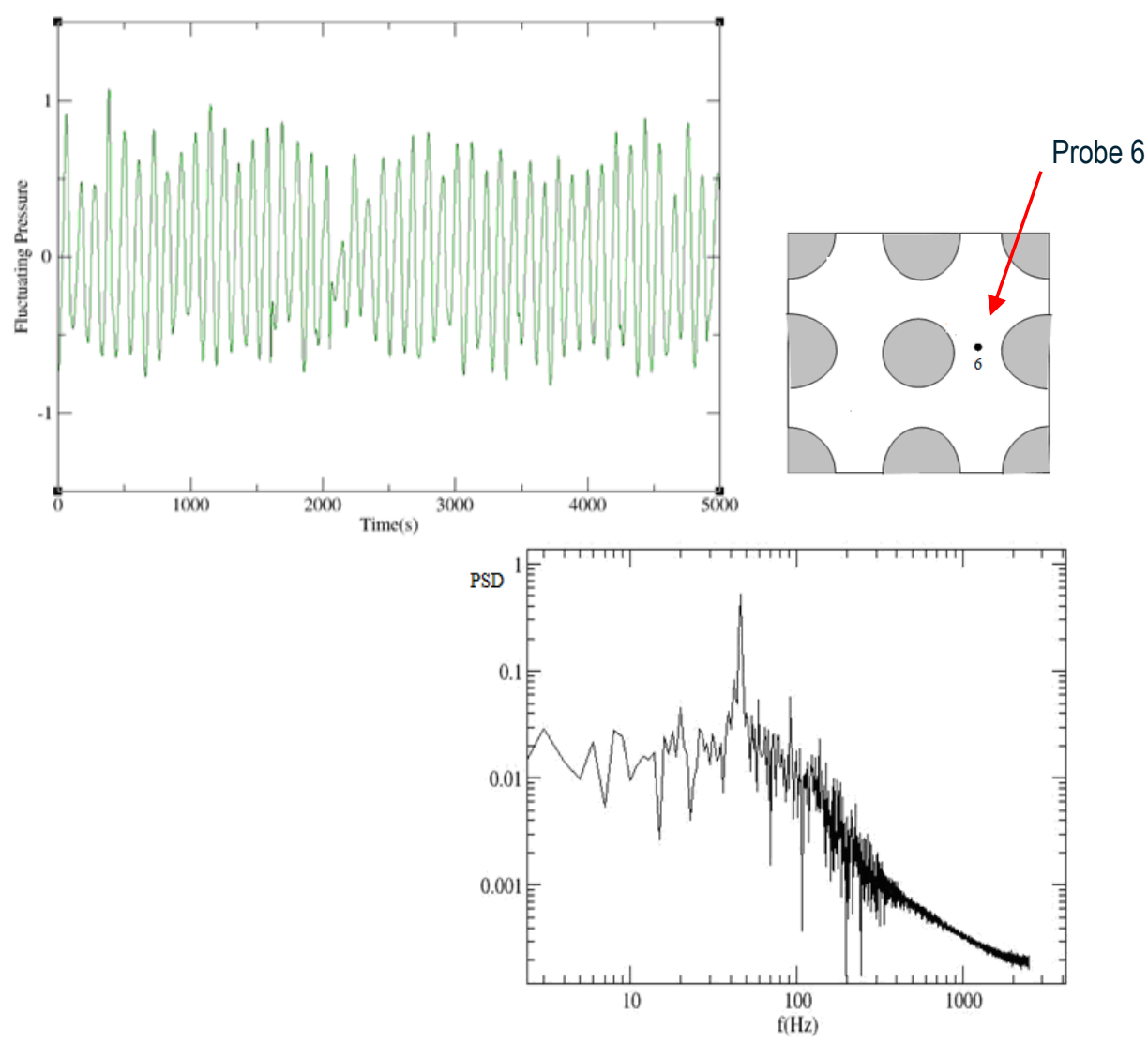

Fig.9. Pressure's signal and its PSD at a wake location (probe 6). Calculated from SST- $C_{a s}$ model.
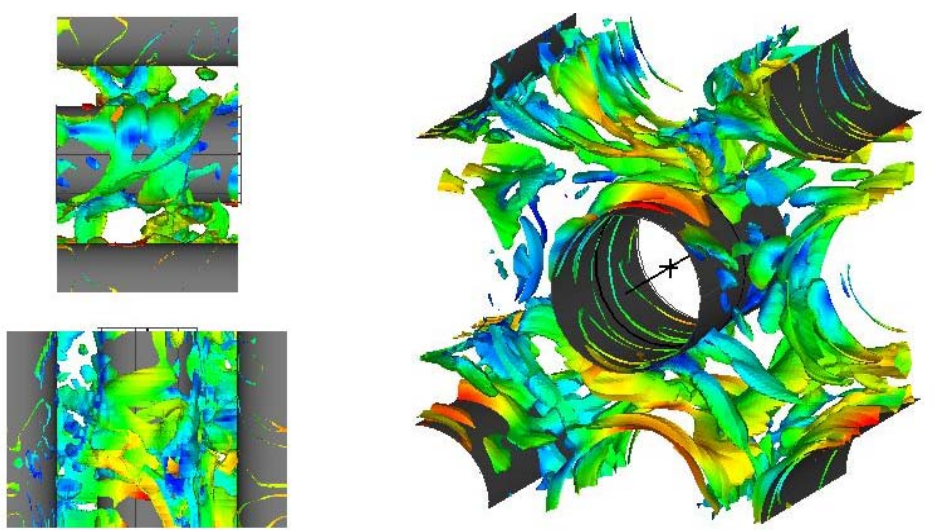

Fig.10. Instantaneous Q Iso-surfaces, results obtained using the SST- $C_{a s}$ model. 